\title{
Wind farm power optimization via yaw angle control: A wind tunnel study
}

Cite as: J. Renewable Sustainable Energy 11, 023301 (2019); https://doi.org/10.1063/1.5077038

Submitted: 24 October 2018 . Accepted: 02 February 2019. Published Online: 08 March 2019

Majid Bastankhah, and Fernando Porté-Agel

\section{COLLECTIONS}

F This paper was selected as Featured
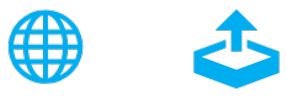

\section{ARTICLES YOU MAY BE INTERESTED IN}

Influence of atmospheric stability on wind-turbine wakes: A large-eddy simulation study Physics of Fluids 27, 035104 (2015); https://doi.org/10.1063/1.4913695

Wind tunnel study of the wind turbine interaction with a boundary-layer flow: Upwind region, turbine performance, and wake region

Physics of Fluids 29, 065105 (2017); https://doi.org/10.1063/1.4984078

Energy extraction properties of a flapping wing with an arc-deformable airfoil Journal of Renewable and Sustainable Energy 11, 023302 (2019); https:// doi.org/10.1063/1.5079864

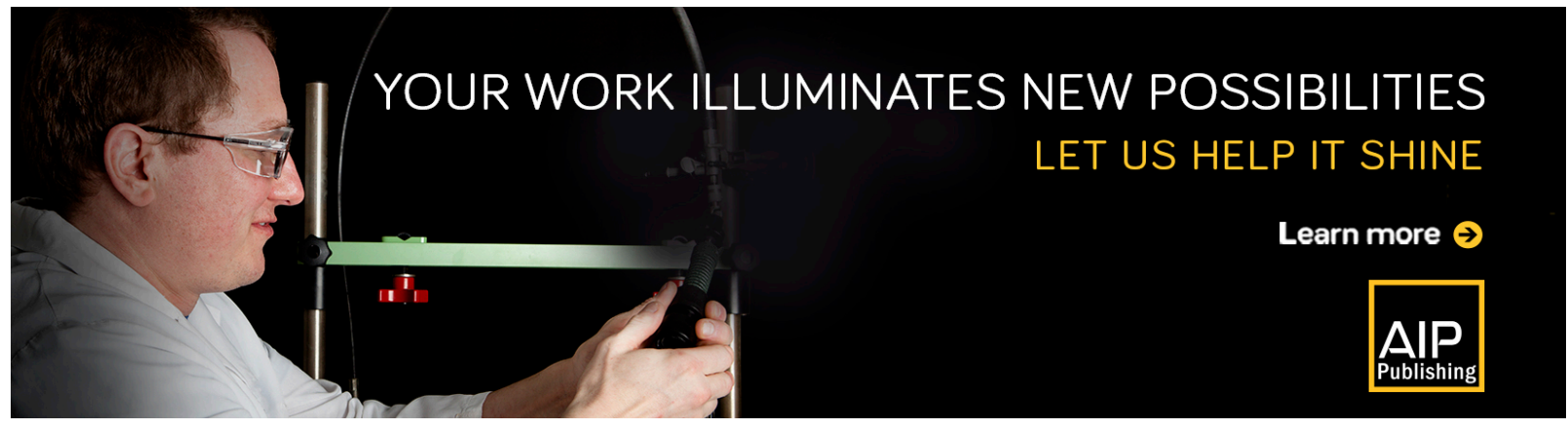




\title{
Wind farm power optimization via yaw angle control: A wind tunnel study
}

\author{
Cite as: J. Renewable Sustainable Energy 11, 023301 (2019); doi: 10.1063/1.5077038 \\ Submitted: 24 October 2018 - Accepted: 2 February 2019 . \\ Published Online: 8 March 2019

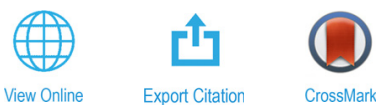

Majid Bastankhah $^{\text {a) }}$ and Fernando Porté-Agel ${ }^{\text {b) }}$

\author{
AFFILIATIONS \\ Wind Engineering and Renewable Energy Laboratory (WiRE), EPFL, Lausanne, Switzerland \\ a) Current address: Department of Engineering, Durham University, Durham, United Kingdom. \\ ${ }^{b)}$ Author to whom correspondence should be addressed: fernando.porte-agel@epfl.ch
}

\begin{abstract}
Yaw angle control is known nowadays as a promising and effective technique to mitigate wake effects in wind farms. In this paper, we perform wind tunnel experiments to study the performance of a model wind farm with five turbine rows under a wide variety of yaw angle distributions. Electrical servo controllers are used to monitor and control the operating conditions of each model wind turbine, which consists of a recently developed, highly efficient rotor with a diameter of $15 \mathrm{~cm}$. Each turbine is used as a sensor to detect its own inflow conditions. Using this method ensures us that all the turbines within the wind farm always operate with an optimal rotational velocity, regardless of their yaw angles or inflow conditions. Wind farm power measurements are carried out for more than 200 cases with different yaw angle distributions. Our results show that yaw angle control can increase the overall wind farm efficiency as much as $17 \%$ with respect to fully non-yawed conditions. Special emphasis is placed on studying yaw angle distributions with different levels of simplicity and power improvement. Among different yaw angle distributions, the most successful ones are those with a relatively large yaw angle value for the first turbine row, and then, the yaw angle decreases progressively for downwind rows until it eventually becomes zero for the last one. In addition, power measurements show that yaw angle control can improve the wind farm efficiency more noticeably for a larger number of turbine rows although this improvement is expected to reach a plateau after several rows.
\end{abstract}

Published under license by AIP Publishing. https://doi.org/10.1063/1.5077038

\section{INTRODUCTION}

Significant power losses due to complex interactions of wind turbine wakes in wind farms call for the development of novel wake control strategies. One approach to achieve this goal that draws increasing attention is the yaw angle control of wind turbines. Yaw angle control is considered to be an effective strategy by deflecting the wakes away from downwind turbines. ${ }^{1,2}$ In this approach, the whole wind farm power production is improved at the expense of reducing the performance of some of the turbines located in the first rows of wind farms.

Wake deflection associated with yawed rotors has been the subject of research since the pioneering studies by Glauert ${ }^{3}$ and Coleman et $\mathrm{al}^{4}$ followed by other studies such as by Grant et $a .^{5}$ and Grant and Parkin. ${ }^{6}$ More information on far wakes of yawed turbines has been, however, provided by more recent studies such as by Medici and Alfredsson, ${ }^{7}$ Jiménez et al., ${ }^{1}$ and Fleming et al. ${ }^{8}$ In particular, several experimental, numerical, and theoretical studies have been carried out in the last three years which were able to report or explain several phenomena associated with yawed turbines and their wake structures. These include, among others, (i) kidney (curled) shape of the wake cross-section due to the presence of a counter-rotating vortex pair (CVP), ${ }^{2,9-13}$ (ii) vertical displacement of yawed turbine wakes due to the interaction of wake rotation, CVP, and ground, ${ }^{2,13}$ (iii) presence of a strong lateral asymmetry in the upwind induction region, ${ }^{14,15}$ (iv) asymmetric distribution of the wake skew angle with respect to the wake center, ${ }^{2}$ and (iv) turbine thrust $\mathrm{t}^{15,16}$ and power ${ }^{15-18}$ variation with the yaw angle. Bartl et al. ${ }^{19}$ have also very recently studied the effect of incoming turbulence and shear on wind turbine wakes in yawed conditions.

Simple analytical models that can predict wakes of yawed turbines have also been developed in the literature. These computationally inexpensive models are useful tools to assess the suitability of yaw angle control for wind farms. Jiménez et al. ${ }^{1}$ used the conservation of mass and momentum to predict the 
wake deflection for yawed turbines by assuming a top-hat shape for the velocity deficit profile. Bastankhah and Porté-Agel ${ }^{2}$ used detailed wind tunnel data to simplify Reynolds-averaged NavierStokes equations, upon which a more realistic analytical model was built. This simple model can predict both streamwise and lateral velocity distributions in the wake of a yawed turbine. More recently, Shapiro et al. ${ }^{12}$ modeled the wake by viewing the yawed turbine as a lifting surface.

The ultimate goal of research on yawed turbines and their wake flows is to successfully implement yaw angle control in wind farms. However, compared to the extensive recent studies on yawed turbines and their wake flows, wind farm power optimization with yaw angle control has received much less attention in the literature. Among a few studies, Adaramola and Krogstad $^{20}$ performed wind tunnel measurements to study the effect of yawing on the performance of two model wind turbines operating in tandem with an inter-turbine spacing of three rotor diameters. They reported an improvement of $12 \%$ in the total power production when the upstream turbine operates with a yaw angle $\gamma$ equal to $30^{\circ}$. It is, however, worth mentioning that the way that they yawed the first turbine created a nonnegligible lateral displacement of the first turbine, which in turn helps further improve the total power production. Ozbay et al. ${ }^{16}$ experimentally studied the performance of two wind turbines, spaced two rotor diameters apart in the streamwise direction, for different values of the upwind turbine yaw angle. While they did not observe any power increase in the case of high incoming turbulence (around 18\% turbulence intensity), the total power for a lower incoming turbulence level (around 8\% turbulence intensity) was found to be improved by $6 \%$, provided that the first turbine's yaw angle $\gamma_{1}$ is equal to $10^{\circ}$. Gebraad et al ${ }^{21}$ later performed large eddy simulation to study the performance of a wind farm consisting of six turbines arranged in three rows and two columns. They observed that yaw angle control can increase wind farm power production up to $13 \%$. In addition, they reported that yaw angle control can reduce loads on most of the wind turbines in the tested wind farm, both yawed turbines and those placed downstream. Park and Law ${ }^{22,23}$ introduced a datadriven approach based on the Bayesian ascent algorithm to optimize the total wind farm power by real-time control of both pitch and yaw angles of wind turbines. Although using this approach allowed them to achieve up to $27 \%$ improvement on the total power produced by four turbines, limited information on optimal yaw angle distributions was provided. Campagnolo et $a .^{24}$ performed wind tunnel experiments to study the performance of three wind turbine models, which were spaced four rotor diameters apart in the streamwise direction and a half rotor diameter apart in the lateral direction. Using a closed-loop wind farm control algorithm, they were able to achieve a $15 \%$ power increase for the whole wind farm. More recently, Fleming et al. $^{25}$ proposed that large-scale structures in wakes of yawed turbines such as CVP can play a role in having more effective strategies to improve the overall performance of wind farms. They also employed large eddy simulations to show that the performance of a wind farm array with four rows of turbines can be improved by more than $12 \%$ by yawing the turbines placed in the first row. Very recently, Bartl et al. ${ }^{26}$ have conducted detailed wind tunnel experiments to study the effects of yaw misalignment on power production and loads of a downstream turbine placed in both full- and partial-wake conditions. They reported that yaw angle control can result in total power improvement within the range of $3.5 \%-11 \%$, depending on the incoming turbulence level and turbine spacing.

Despite the merit of the above-mentioned studies, they mostly focused on the amount of increase in wind farm efficiency achieved by yaw angle control, with less attention to yaw angle distributions that can lead to such an increase. In the present work, we carried out detailed wind tunnel experiments to study the performance of a wind farm with five rows of model wind turbines for a wide range of yaw angle distributions. These measurements provide new insights into the usefulness of yaw angle control for wind farms with different sizes and yaw angle distributions.

The remainder of this paper is organized as follows. In Sec. II, basic information about the wind tunnel and flow conditions is provided. Section III concerns the characteristics of the model wind turbine employed in this study. The wind farm setup is explained in detail in Sec. IV, and the results are presented in Sec. V. Finally, Sec. VI concludes this paper with a discussion of key findings and future research directions.

\section{WIND TUNNEL AND INFLOW CHARACTERIZATION}

Measurements were performed in the atmospheric boundary-layer wind tunnel (ABLWT) of the WiRE laboratory at EPFL, Switzerland. The length, width, and height of the wind tunnel test section are $28,2.5$, and $2.25 \mathrm{~m}$, respectively. See the previous studies of the authors (e.g., Ref. 15) for more information on the WiRE wind tunnel. Due to the large length of the test section, a turbulent boundary layer is naturally developed over the test-section floor, without the use of any tripping mechanism.

Velocity measurements were first performed to ensure that the turbulent boundary layer is fully developed within the studied wind farm. Prior to the installation of model wind turbines, hot-wire measurements were carried out to quantify vertical profiles of the boundary-layer velocity at the streamwise positions of the wind turbines. The hot-wire anemometer was calibrated against a velocity calibrator from Dantec at the beginning as well as the end of measurements. At each streamwise position, the measurements were performed over a vertical profile starting at $z=1 \mathrm{~cm}$ up to $z=45 \mathrm{~cm}$, where $z$ denotes the vertical distance from the test-section floor. At each measured point, the hot-wire probe consisting of a single $5.0 \mu \mathrm{m}$ tungsten wire collected the data at a rate of $20000 \mathrm{~Hz}$ for a period of $1 \mathrm{~min}$. Figure 1(a) shows vertical profiles of the mean streamwise velocity $\bar{u}$ normalized with the velocity at the turbine hub height $\bar{u}_{h}$ at streamwise positions of the wind turbines, where the overbar denotes time averaging. In the figure, the height is normalized with the rotor diameter $d$, and the turbine hub height $z_{h}$ is shown with a horizontal dashed blue line. T1, T2,..., T5 in the figure stand for model turbines located at different rows; T1 is the most upwind turbine, while $\mathrm{T} 5$ is the most downwind one. The vertical 


\begin{tabular}{|llllllllll|}
\hline$\circ$ & $\mathrm{T} 1$ & $\times$ & $\mathrm{T} 2$ & $\diamond$ & $\mathrm{T} 3$ & $\circ$ & $\mathrm{T} 4$ & $\Delta$ & $\mathrm{T} 5$ \\
\hline
\end{tabular}

(a)

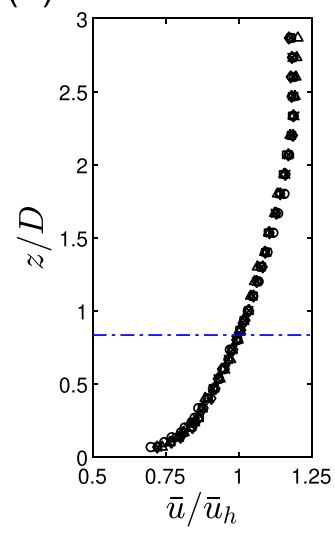

(b)

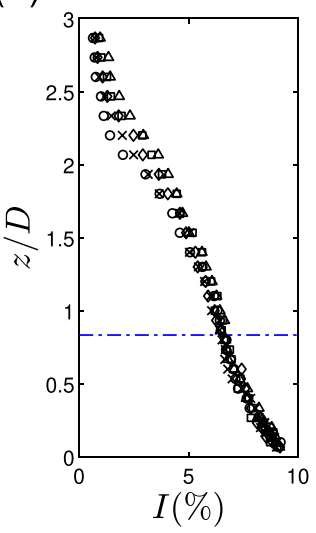

(c)

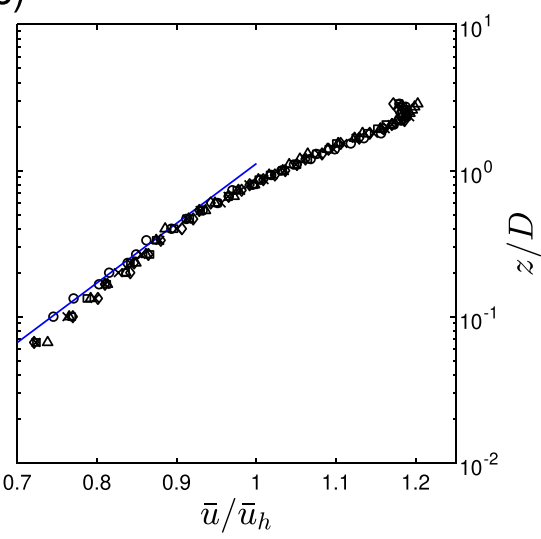

FIG. 1. Characteristics of the turbulent boundary layer flow at the wind turbine positions, prior to the installation of turbines: (a) the normalized mean streamwise velocity profile and (b) the streamwise turbulence intensity profile. The horizontal dotted-dashed lines indicate the turbine hub height. (c) The normalized mean streamwise velocity profile on a semi-logarithmic scale. The solid line shows the fitted logarithmic profile to the velocity profile in the surface layer at $\mathrm{T} 1$. profiles of the streamwise turbulence intensity $\mathrm{I}=\sigma_{u} / \bar{u}_{h}$ are also shown in Fig. 1(b), where $\sigma_{u}$ denotes the standard deviation of the streamwise velocity. As seen in Fig. 1, overall, there is no large variation in the boundary-layer profile within the wind farm, especially at the turbine hub height.

Table I summarizes the key information about the boundary layer approaching the wind farm. The values of the friction velocity $u_{*}$ and the aerodynamic surface roughness length $z_{0}$ for the boundary layer are estimated based on fitting a logarithmic profile to the measured points in the surface layer at $\mathrm{T} 1$ [Fig. 1(c)]. The ratio of the boundary-layer thickness to the turbine rotor diameter $\delta / D$ along with the value of the streamwise turbulence intensity at hub height I $\left(z=z_{h}\right)$ suggests that the current wind tunnel setup simulates a relatively smooth and shallow boundary layer which mostly occurs in offshore cases or those over lands with stable thermal stratification. ${ }^{27,28}$

\section{MODEL WIND TURBINE}

The model wind turbine employed in this wind tunnel study was developed in the previous studies of the authors. ${ }^{29,30}$ It is a three-bladed horizontal-axis miniature wind turbine with a rotor diameter D of $15 \mathrm{~cm}$ and a hub height $z_{h}$ of $12.5 \mathrm{~cm}$. Given its small size, this turbine is especially suitable for wind tunnel simulations of large wind farms. The other advantage of the employed model turbine is the fact that, despite its small size, its thrust $C_{T}$ and power $C_{P}$ coefficients are relatively close to those of utility-scale turbines, which leads to more realistic wind tunnel simulations compared to previous studies. The interested reader is referred to Ref. 29 for detailed information on the turbine model.

A small permanent-magnet DC generator called DCX10L from Maxon is attached to the rotor to convert the mechanical

TABLE I. Key characteristics of the incoming turbulent boundary layer.

\begin{tabular}{ccccc}
\hline \hline$\delta / D$ & $\bar{u}_{h}(\mathrm{~m} / \mathrm{s})$ & $I\left(z=z_{h}\right)$ & $u_{*} / \bar{u}_{h}$ & $z_{0} / D$ \\
\hline 3.2 & 4.8 & $7 \%$ & 0.043 & $9.3 \times 10^{-5}$ \\
\hline \hline
\end{tabular}

power extracted from the incoming wind to the electrical one. The generator is equipped with a digital incremental encoder with an accuracy of 128 counts per turn to measure the instantaneous rotational velocity $\Omega$. A servo-electric controller (henceforth referred to as the RPM controller) is connected to each DC generator to simultaneously monitor and control the value of the rotational velocity $\Omega$ as well as the electrical current $i$ generated by the DC machine. We then use torque calibration of the DC machine performed in the study by Bastankhah and PortéAgel $^{29}$ to estimate the shaft torque $\mathrm{T}$ and consequently the mechanical power (i.e., $P_{\text {mech }}=\mathrm{T} \cdot \Omega$ ) based on the values of $i$ and $\Omega$. The bottom of each turbine tower is also attached to a DC motor. This motor is connected to an angular position controller (henceforth referred to as the yaw angle controller) so that the yaw angle value of each turbine can be controlled individually. Any change in the yaw angle is performed by the controller with an accuracy of $0.1^{\circ}$ although the uncertainty associated with positioning the turbine in the reference case with a zero yaw angle is expected to be around $1^{\circ}$.

Figure 2 shows the mechanical power $P$ of the most upwind turbine (i.e., T1) as a function of the rotational velocity $\Omega$ for different incoming velocities and yaw angles. As expected, the turbine power decreases with a decrease in the incoming velocity as well as an increase in the yaw angle. For each case, values of the free-rotating rotational velocity $\Omega_{f}$ and the optimal one $\Omega_{o}$ (i.e., corresponding to the turbine maximum power) are shown in the figure with filled and hollow circles, respectively. Even though wind farm experiments were performed for a constant incoming velocity $\left(\bar{u}_{h}=4.8 \mathrm{~m} / \mathrm{s}\right)$, the information provided in Fig. 2 will be used in Sec. IV to ensure that all the turbines within the wind farm always operate at the optimal rotational velocity. Note that we did not seek the $\mathrm{P}-\Omega$ relationship for values of $\Omega$ much lower than $\Omega_{0}$, as the goal in this study is only to find the corresponding values of $\Omega_{f}$ and $\Omega_{o}$ for a range of $\bar{u}$ and $\gamma$ values, as elaborated later in Sec. IV. Detailed information on the variation of power and thrust force of this model wind turbine with the rotational velocity and the yaw angle can be found in Ref. 15. 


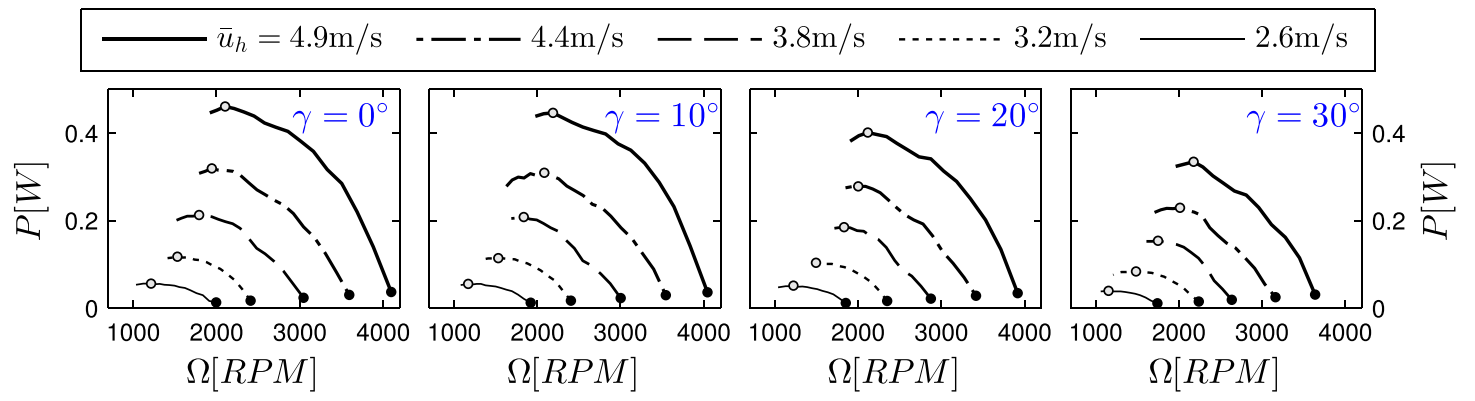

FIG. 2. Variation of the first turbine's power with rotational velocity for different values of incoming velocity and yaw angle.

\section{WIND FARM SETUP}

The wind tunnel setup consists of a column of five wind turbines in a line parallel to the incoming flow direction as schematically shown in Fig. 3. The inter-turbine spacing is five rotor diameters, which lies in the range of the spacing between utility-scale wind turbines in the field. ${ }^{31}$ During measurements, as mentioned in Sec. III, both the rotational velocity and the yaw angle of the turbines are controlled with servo controllers as shown in Fig. 3. Note that we do not change the yaw angle of the last turbine (T5) as it is less likely to result in any wind farm power improvement.

A homemade LabVIEW code is used to enable communications between a Data Acquisition (DAQ) hardware from National Instruments and all the controllers. The DAQ system sends the required information such as desired values of the yaw angle $\gamma$ and the rotational velocity $\Omega$ to the controllers, and it receives the data on instantaneous values of the rotational velocity $\Omega$ as well as the electrical current $i$ for each turbine (see Fig. 3).

For studies on yaw angle control in wind farms, it is crucial to ensure that the power variation measured between scenarios with different yaw angle distributions is only due to the change of the turbines' yaw angles and not because of operation of turbines with non-optimal rotational velocities. In general, the optimal rotational velocity $\Omega_{o}$ of a turbine depends on its inflow conditions and the turbine yaw angle. As a result, any change in the yaw angle of either upwind turbines or the turbine itself necessitates the readjustment of the turbine rotational velocity. Figure 4 illustrates the method implemented in this study to ensure that all the turbines operate at $\Omega_{0}$ for any given yaw angle distribution. In the first step, a priori power measurements for a single turbine are performed to determine $\Omega_{0}$ for different incoming velocities and yaw angles, similar to those reported in

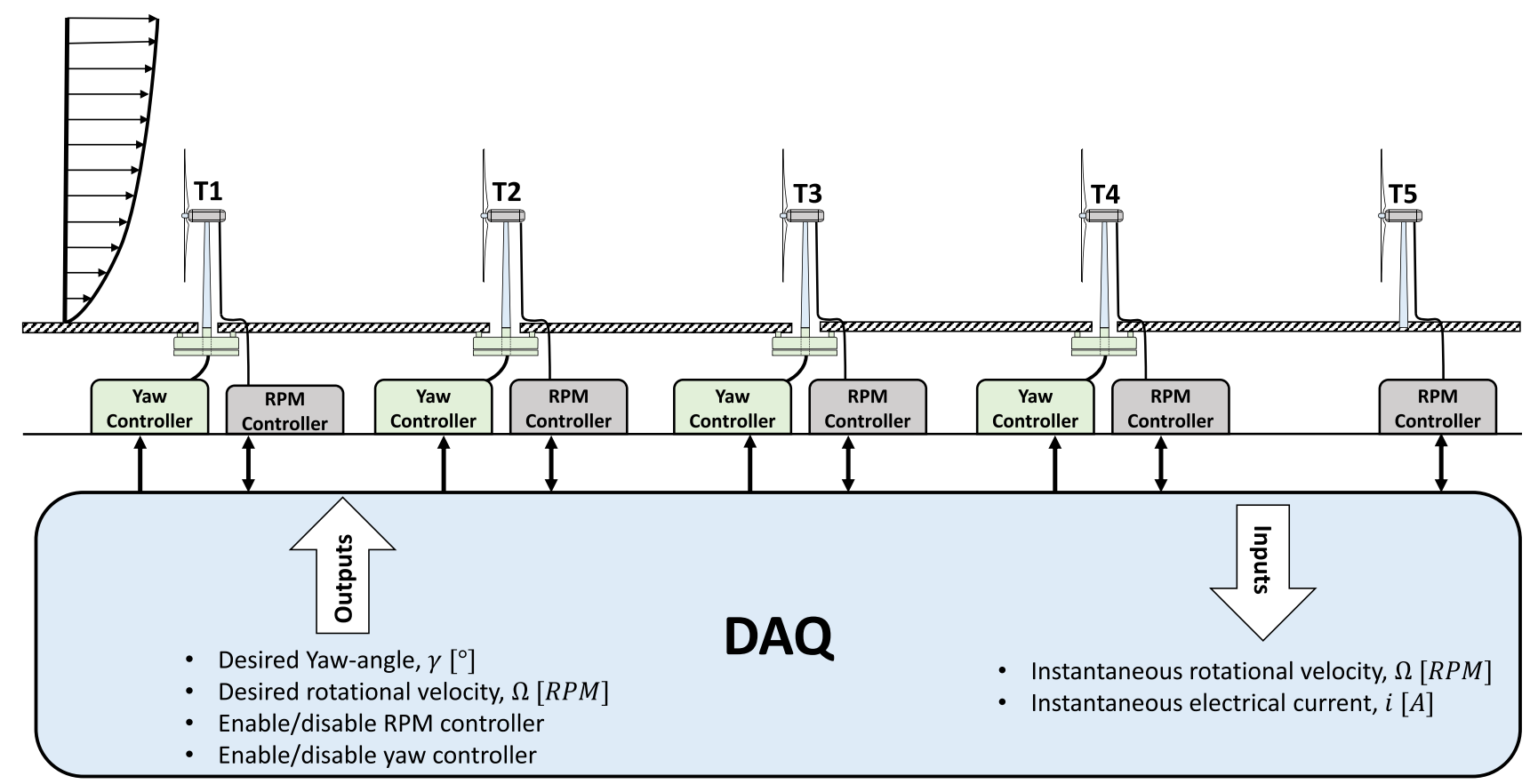

FIG. 3. Schematic figure of the wind farm setup including the model wind turbines, the electrical controllers, and the DAQ system. 


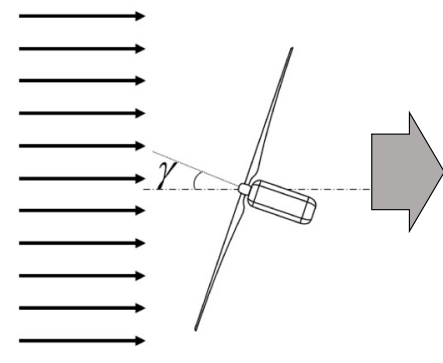

1

Power of a single wind turbine is measured for different incoming velocities and yaw angles.

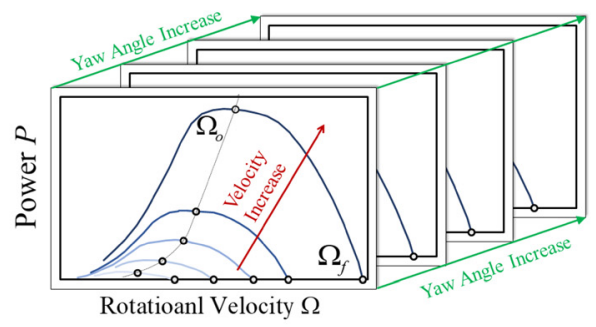

2

For each case, values of free-rotating
rotational velocity $\Omega_{f}$ and optimal
rotational velocity $\Omega_{o}$ are found.

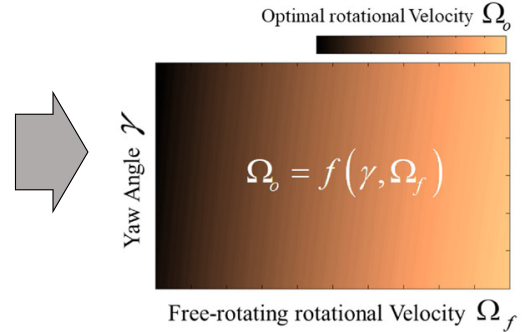

3

Optimal rotational velocity $\Omega_{o}$ for any given values of yaw angle $\gamma$ and freerotating rotational velocity $\Omega_{f}$ is estimated.

FIG. 4. Schematic figure showing the method to find the optimal rotational velocity $\Omega_{0}$ for the wind turbines within the wind farm operating at any given yaw angle and inflow condition.

Fig. 2. Therefore, for any given pair of known yaw angle $\gamma$ and measured free-rotating rotational velocity $\Omega_{f}$, the optimal rotational velocity $\Omega_{0}$ can be estimated by interpolating the available data, stored in a lookup table (i.e., $\Omega_{o}=f\left(\gamma, \Omega_{f}\right)$ ). As a result, for a given wind farm yaw angle distribution, the value of $\Omega_{0}$ for each turbine with a known $\gamma$ can be estimated by measuring $\Omega_{f}$ for a few seconds. This method is particularly useful for small-size model turbines since by measuring $\Omega_{f}$, each turbine acts as a sensor estimating its own incoming flow conditions. For largescale turbines, with the possibility of having a velocity sensor on the turbine nacelle, the value of $\Omega_{o}$ can be directly determined from velocity measurements (i.e., $\Omega_{o}=f\left(\gamma, \bar{u}_{h}\right)$ ).

The operating conditions of turbines in the wind farm are set sequentially. This means that, for each scenario, the procedure starts by setting $\gamma$ and $\Omega_{0}$ of the first turbine (T1), and it continues for downwind turbines one after the other until all of them are set. Setting turbine operating conditions in a sequential order is important as $\Omega_{0}$ for each turbine should be estimated only when all upwind turbines operate with the desired values of $\gamma$ and $\Omega$. For the sake of saving time, $\gamma$ for the $i$ th turbine in the $j$ th scenario is set only if it is different from the corresponding value in the previous scenario (i.e., $\gamma_{i}^{j} \neq \gamma_{i}^{(j-1)}$ ), where the superscript $j$ denotes the scenario number ranging from 1 to $m$ and the subscript $i$ denotes the turbine row number ranging from 1 to $n(n=5$ in this study). Likewise, $\Omega_{o}$ of the $i$ th turbine in the jth scenario is set only if yaw angle values of the $i$ th turbine and upwind turbines are not the same as those in the previous scenario (i.e., $\left\{\gamma_{1}^{j}, \ldots, \gamma_{i}^{j}\right\} \neq\left\{\gamma_{1}^{(j-1)}\right.$, $\left.\left.\ldots, \gamma_{i}^{(j-1)}\right\}\right)$. Once the adjustment of all the turbines is finished for each scenario, the power extracted by the turbines is measured and recorded. The interested reader is referred to the Appendix where a flowchart of the whole process concerning adjustment and measurement of the turbines' operating conditions is depicted.

\section{WIND FARM POWER MEASUREMENTS}

In this section, we present wind farm power measurements for different yaw-angle distributions. Except for the last turbine
(T5), we vary the yaw angle of all the turbines from $\gamma_{\text {min }}=0^{\circ}$ to $\gamma_{\max }=30^{\circ}$ with a step of $5^{\circ}$. For the sake of simplicity, the turbines are only yawed in the counterclockwise direction, seen from the top, in this study. We also exclude less promising scenarios by considering only the ones at which the yaw angles of turbines are always equal to or greater than those of their downwind turbines. This can be explained by bearing in mind that yawing turbines can improve the wind farm power production by steering wakes away from downwind turbines. Therefore, yaw misalignment for upwind turbines is more likely to be advantageous as, in this case, there are more turbines placed downstream that can benefit from this situation. Given the adopted testing strategies, the total number of scenarios is computed in Table II for different numbers of turbine rows and yaw angles. The variable $q$ is the number of tested yaw angles which is equal to $\left(\gamma_{\max }-\gamma_{\min }\right) /$ step +1 . Note that, given the trend observed in Table II, one can easily compute the total number of scenarios for wind farms with more than five rows.

In a brute-force approach, we carried out wind farm power measurements for the whole 210 scenarios to test all different

TABLE II. Total number of scenarios with different yaw angle distributions. The variable $q$ is the number of tested yaw angles which is equal to $\left(\gamma_{\max }-\gamma_{\min }\right) / \mathrm{step}+1$.

\begin{tabular}{lcc}
\hline \hline \multirow{2}{*}{ Number of turbine rows, $n$} & \multicolumn{2}{c}{ Number of scenarios, $m$} \\
\hline 2 & Mathematical formulation & Current setup $(q=7)$ \\
3 & $\sum_{i=1}^{q} i$ & 7 \\
4 & $\sum_{j=1}^{q} \sum_{i=1}^{j} i$ & 28 \\
5 & $\sum_{k=1}^{q} \sum_{j=1}^{k} \sum_{i=1}^{j} i$ & 84 \\
\hline \hline
\end{tabular}




\begin{tabular}{|lllllllllll|}
\hline$\cdot \gamma=0^{\circ}$ & $\cdot$ & $5^{\circ}$ & $10^{\circ}$ & $15^{\circ}$ & $\circ$ & $20^{\circ}$ & $\bullet$ & $25^{\circ}$ & $\bullet$ & $30^{\circ}$ \\
\hline
\end{tabular}

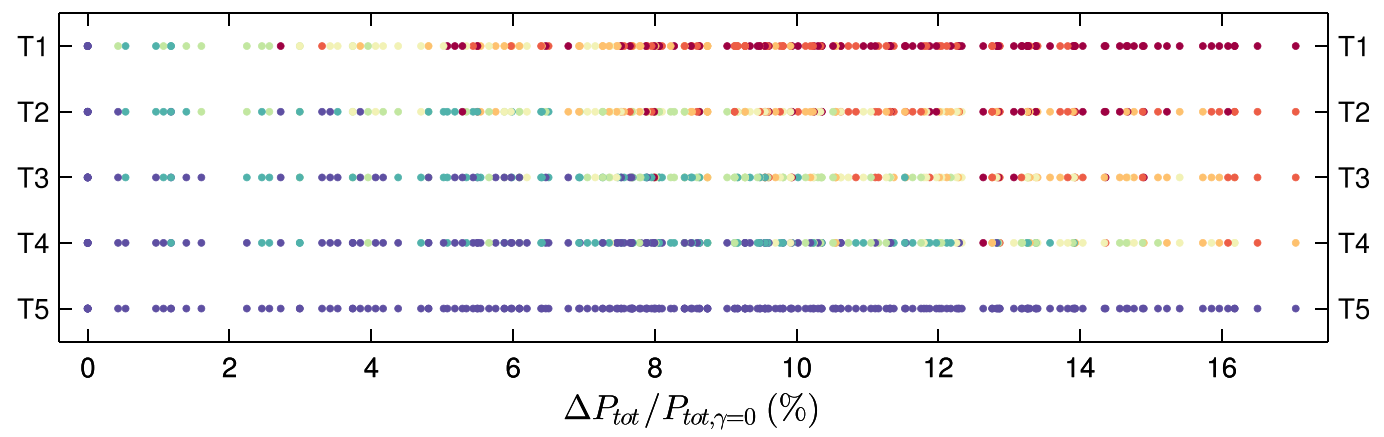

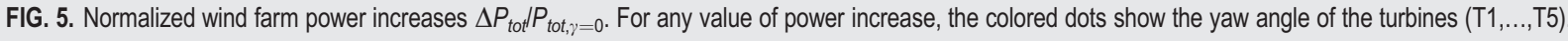

yaw angle distributions that can potentially improve the wind farm efficiency. For each scenario, the adjustment of turbines' operating conditions lasted from one to three minutes, and power measurements were then carried out for one minute with the sampling frequency of $2000 \mathrm{~Hz}$.

All the different yaw angle distributions examined in this paper result in an increase in the wind farm power output (i.e., $\Delta P_{\text {tot }}>0$ ) with respect to the reference case $P_{\text {tot }, \gamma=0}$ (i.e., nonyawed conditions), where $P_{\text {tot }}=\sum_{i=1}^{n} P_{i}$. Figure 5 shows the normalized wind farm power increase $\Delta P_{\text {tot }} / P_{\text {tot }, \gamma=0}$ as well as the yaw angle distribution for all the tested scenarios. For each case, the value of power increase is indicated on the plot's horizontal axis. The colored dots in the plot area represent the corresponding yaw angle values for turbines at each row, specified on the plot's vertical axis. Note that each color corresponds to a given yaw angle value shown in the plot's legend. As seen in the figure, the normalized wind farm power increase varies from $0.5 \%$ to $17 \%$ depending on the yaw angle distribution. In the following, we employ the available wind tunnel data to examine three different strategies to implement yaw angle control, with different levels of complexity and power improvement. We

(a)

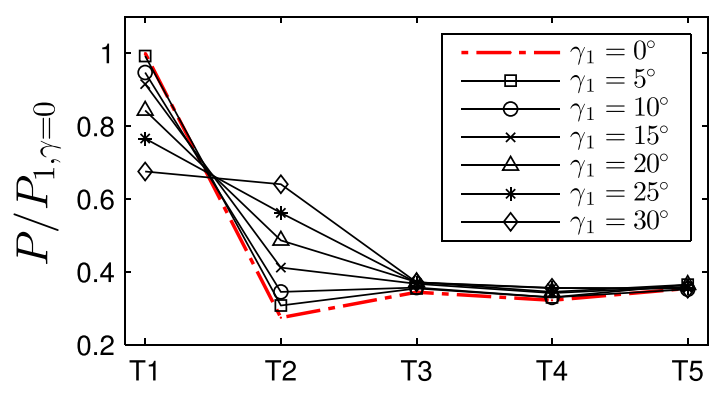

believe that this categorization can be useful in real applications, for which the most suitable scenarios in terms of power production might be ruled out due to other constraints associated with structural loads or the wind turbine yaw mechanism.

- Strategy I: As the simplest approach, only the most upstream turbine (T1) is yawed (i.e., $\gamma_{1} \neq 0, \gamma_{2: 5}=0$ ). Figure 6(a) shows the normalized power extracted by the turbines for different values of $\gamma_{1}$. The figure shows that the second turbine's power $P_{2}$ is significantly increased by yawing the first turbine (e.g., $P_{2}$ for $\gamma_{1}=30^{\circ}$ is more than double the one for $\gamma_{1}=0^{\circ}$ ). The effect of $\gamma_{1}$ on the turbines in the third row and further downstream is indeed less considerable. Despite the simplicity of implementing this strategy for wind farm operators, Fig. 6(b) shows that the resultant wind farm power improvement is much smaller than the maximum achievable value shown by a dashed line.

- Strategy II: In this strategy, all the turbines (except for the last one) have the same non-zero yaw angle (i.e., $\gamma_{1}=\gamma_{2}=\gamma_{3}$ $=\gamma_{4} \neq 0$ and $\gamma_{5}=0$ ). Figure 7 (a) shows the normalized power distribution for different values of $\gamma_{1: 4}$. As seen in the figure, one can observe a power increase for T2, T3, and T4

(b)

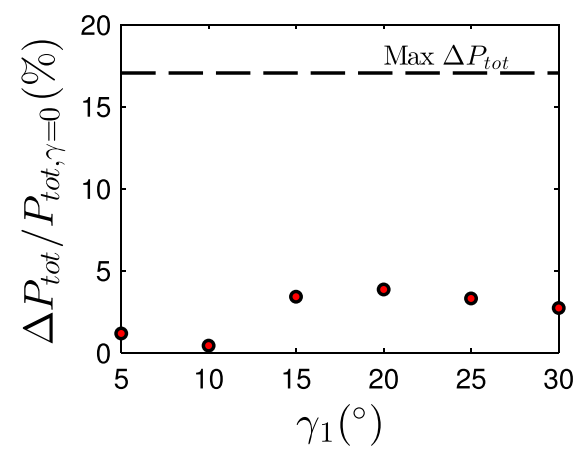

FIG. 6. (a) Normalized power distribution for different values of $\gamma_{1}$ when $\gamma_{2: 5}=0$. Power values are normalized with the one of T1 with a zero yaw angle. The red dashed curve shows the power distribution for fully non-yawed conditions $\left(\gamma_{1: 5}=0\right)$. (b) Normalized total wind farm power increase (\%) for different values of $\gamma_{1}$. The black dashed line shows the maximum wind farm power increase achieved by yaw angle control. 
(a)

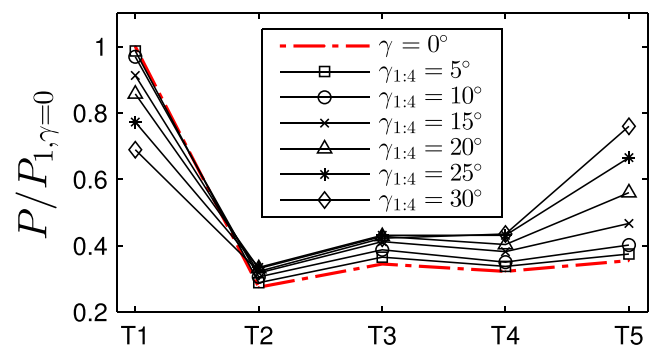

(b)

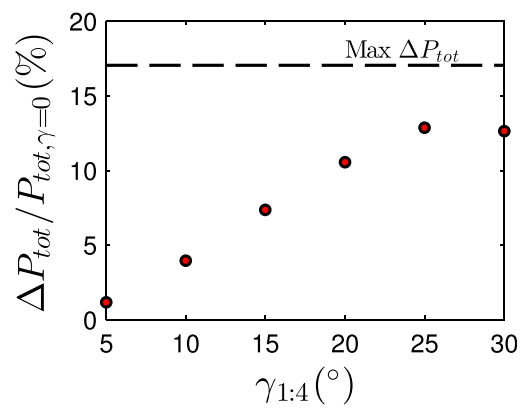

FIG. 7. (a) Normalized power distribution when all the first four turbines have the same non-zero yaw angle (i.e., $\gamma_{1}=\gamma_{2}=$ $\gamma_{3}=\gamma_{4} \neq 0$ and $\gamma_{5}=0$ ). The values of turbine powers are normalized with the one of T1 with the zero yaw angle. The red dashed curve shows the power distribution for fully non-yawed conditions $\left(\gamma_{1: 5}=0\right)$. (b) Normalized total wind farm power increase (\%) for different values of $\gamma_{1: 4}$. The black dashed line shows the maximum wind farm power increase achieved by yaw angle control. although all of them operate with a non-zero yaw angle. This can be explained by the fact that their power reduction due to yawed conditions is compensated by more favorable inflow conditions since they are less affected by deflected wakes of upwind turbines. However, the major power improvement occurs for the last turbine as it is still a nonyawed turbine with a much more favorable inflow condition compared to that of the reference case. Figure 7(b) shows that the power increase occurred for those placed downwind of the first turbine considerably outweighs the power reduction of the first turbine. This strategy increases the total wind farm power more than $12 \%$ for those with $\gamma_{1: 4}$ $=25^{\circ}$ or $30^{\circ}$. Even though the results suggest that this strategy can considerably improve the wind farm power efficiency, it is still not among the most successful scenarios tested in this study. In the following, we focus on those that yield the maximum power improvement.

- Strategy III: We do not impose any limitation on yaw angle values within this approach. Instead, we investigate the most effective yaw angle distributions in terms of wind farm efficiency (i.e., $\gamma_{1: 4} \geq 0$ and $\gamma_{5}=0$ ). Figure 8 shows the normalized power extracted by the turbines for the top decile of scenarios when ranked by the magnitude of $\Delta \mathrm{P}_{\text {tot }}$. Moreover, the yaw angle distributions corresponding to these scenarios are shown in this figure. The mean values of both power and yaw angle distributions are shown with black curves in the figure, where the error bars indicate the standard deviation. Moreover, similar to previous figures, the red dashed curve in the figure acts as a reference by showing the power distribution for fully non-yawed conditions. Yaw angle distributions shown in the figure enhance the overall wind farm power by $14 \%-17 \%$. In comparison with the reference case, one can observe that the power distribution within the wind farm is much more homogeneous for the most effective scenarios. The figure also shows that, on average, this more uniform power distribution is achieved by having a large yaw angle for the first turbine and smaller values for downwind turbines. The figure suggests that the optimal yaw angle distribution can be roughly estimated by a linear relationship varying from the maximum value for the first turbine to zero for the last one.

The available data are also used to shed light on how the profitability of yaw angle control in terms of power production varies with the number of wind turbine rows. In total, 84, 28, and 7 different scenarios are tested to examine the effect of yaw angle control on the power of wind farms with 4,3 , and 2 turbine rows, respectively.

Figures 9 and 10 show the normalized power and yaw angle distribution which corresponds to the top decile of scenarios for four and three turbine rows, respectively. The maximum total power enhancement via yaw angle control for four turbines is about $13 \%$, while this value is reduced to $8 \%$ for three rows. As shown in Figs. 9 and 10, the optimum yaw angle distributions are again those with a large $\gamma_{1}$ and smaller yaw angle values for downwind turbines. This is consistent with the data reported by Campagnolo et $a l^{24}$ It is also worth noting that the optimum value of $\gamma_{1}$ decreases with the decrease in the number of turbine rows (compare $\gamma_{1}$ in Figs. 9 and 10). Figure 11(a) shows the effect of yawing on the performance of two turbines. Although all the tested yaw angles from $5^{\circ}$ to $30^{\circ}$ result in a total power increase, the maximum power improvement is only $3.6 \%$ and it occurs for $\gamma_{1}$ equal to $20^{\circ}$.
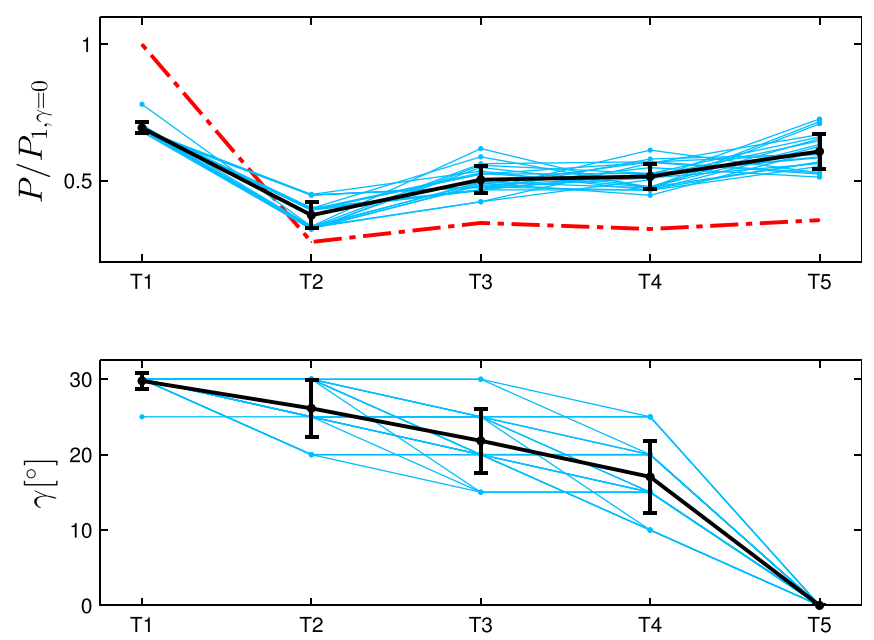

FIG. 8. Distributions of power and yaw angles for the top decile of the tested scenarios when ranked by the magnitude of $\Delta P_{\text {tot }}$. The values of turbine powers are normalized with the one of T1 with the zero yaw angle. Black curves show the mean values, and the error bars indicate the standard deviation. The red dashed curve shows the power distribution for fully non-yawed conditions $\left(\gamma_{1: 5}=0\right)$. 

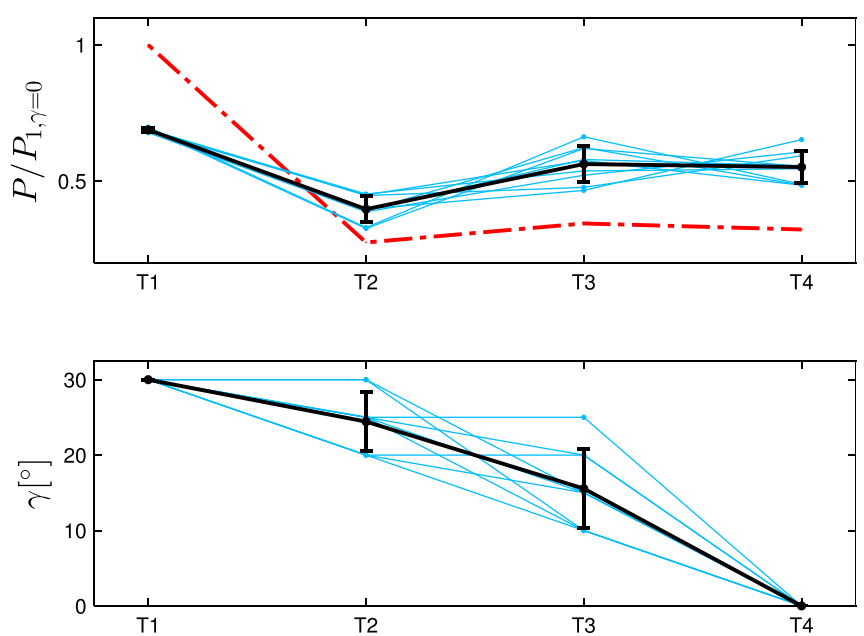

FIG. 9. Power and yaw angle distributions of four turbine rows for the top decile of the tested scenarios when ranked by the magnitude of $\Delta P_{\text {tot. }}$. The values of turbine powers are normalized with the one of T1 with the zero yaw angle. Black curves show the mean values, and the error bars indicate the standard deviation. The red dashed curve shows the power distribution for fully non-yawed conditions $\left(\gamma_{1: 4}=0\right)$.

In the following, we employ the analytical wake model, developed in the previous work of the authors, ${ }^{2}$ to provide an estimation on how the presented results are related to other operating conditions. In particular, we are interested to examine the performance of two wind turbines as a function of the first turbine yaw angle $\gamma_{1}$ for different values of incoming turbulence intensity I and streamwise turbine spacing $S_{x}$. To estimate the incoming velocity for the second (i.e., downstream) turbine, the analytical model is used to compute the wind speed averaged over its rotor area. The wake recovery rate $k$, the model input, is obtained based on the empirical formula: ${ }^{32} k=0.35 \mathrm{I}$, and the value of the turbine thrust coefficient $C_{T}$ is obtained from the previous work of the authors ${ }^{15}$ for a similar turbine. The power extracted by upstream and downstream turbines can be then estimated from Fig. 2, given the fact that the incoming velocity and the yaw angle are known for both of them. Figures 11(b) and 11(c) show the model predictions of the normalized total power increase for different values of $S_{x}$ and $I(\%)$, respectively. Note that the value of I in Fig. 11(b) is equal to 7\% for all the shown cases, while the value of $S_{x}$ is equal to $5 D$ for all the cases shown in Fig. 11(c). This means that the solid lines in the figures show model predictions for conditions similar to those in the current setup.

Figure 11(b) shows that the yaw angle control becomes less effective for larger values of $S_{x}$. This may seem surprising at the first glance since the deflection of the upwind turbine wake increases with the increase in the streamwise distance. This can be explained by noting that although the wake deflection for a yawed turbine increases rapidly with the streamwise distance at first (e.g., $x<4 \mathrm{D}$ ), its variation becomes slower as the wake moves further downstream. ${ }^{1,2}$ More importantly, for cases with larger values of $S_{x}$, the second turbine is basically less affected by the wake of the upstream turbine, which in turn limits the usefulness of any wake mitigation strategy including yaw angle control. Figure 11(b) also shows that the optimum yaw angle decreases with the increase in $S_{x}$.

In addition to turbine spacing, Fig. 11(c) shows that the incoming turbulence intensity plays an important role in relation to the usefulness of yaw angle control. This is mainly due to the fact that a higher turbulence level enhances flow entrainment and thereby faster wake recovery. This means that, in highly turbulent inflow conditions, there is less room for wake mitigation strategies to improve the performance of downwind turbines. ${ }^{33}$

In the following, we examine the effect of the wind farm size on the suitability of yaw angle control in more detail. Figure 12(a) shows the maximum wind farm power increase, obtained with yaw angle control, as a function of number of turbine rows $n$. One can observe that the implementation of yaw angle control is clearly more advantageous for larger wind farms. In fact, Fig. 12(a) shows that the wind farm power increase varies linearly with the number of turbine rows. It is of interest to see if the linear trend shown in this figure can be maintained for larger wind farms. Within this line of thought, it might be more informative to use a new metric to quantify the wind farm performance as shown in Fig. 12(b). Here, the wind farm efficiency $\eta$ is defined as the total wind farm power (i.e., $P_{\text {tot }}$ ) divided by the power extracted by the same number of turbines under undisturbed inflow conditions (i.e., $\left.\left(n \times P_{1, \gamma=0}\right)\right)$. Figure 12(b) shows that the wind farm with yaw angle control has higher efficiency than the non-yawed wind farm, and the difference between the two cases becomes broader with the increase in $n$. This is consistent with the results already reported in Fig. 12(a). However, by extrapolating the variation of $\eta$ for values of $n$ larger than 5 , one can suggest that the wind farm is likely to reach asymptotic conditions after a few more rows, where $\eta$ has a constant value (a)

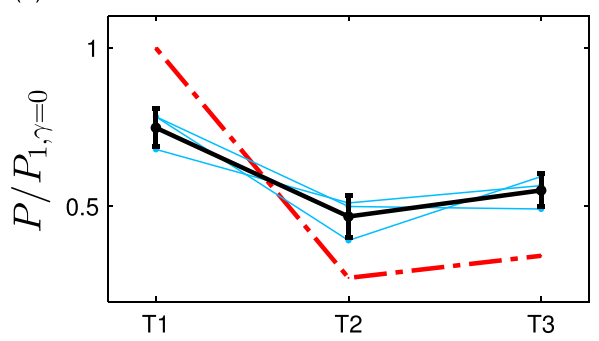

(b)

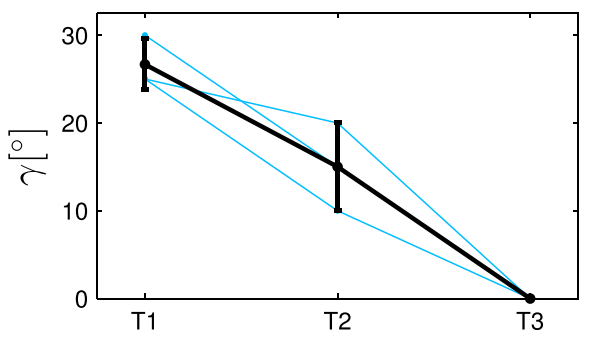

FIG. 10. Power and yaw angle distributions of three turbine rows for the top decile of the tested scenarios when ranked by the magnitude of $\Delta P_{\text {tot }}$. The values of turbine powers are normalized with the one of T1 with the zero yaw angle. Black curves show the mean values, and the error bars indicate the standard deviation. The red dashed curve shows the power distribution for fully non-yawed conditions $\left(\gamma_{1: 3}=0\right)$. 
(a)

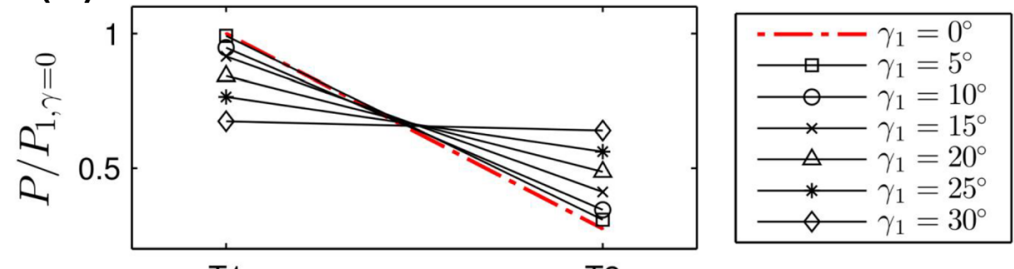

T1

T2
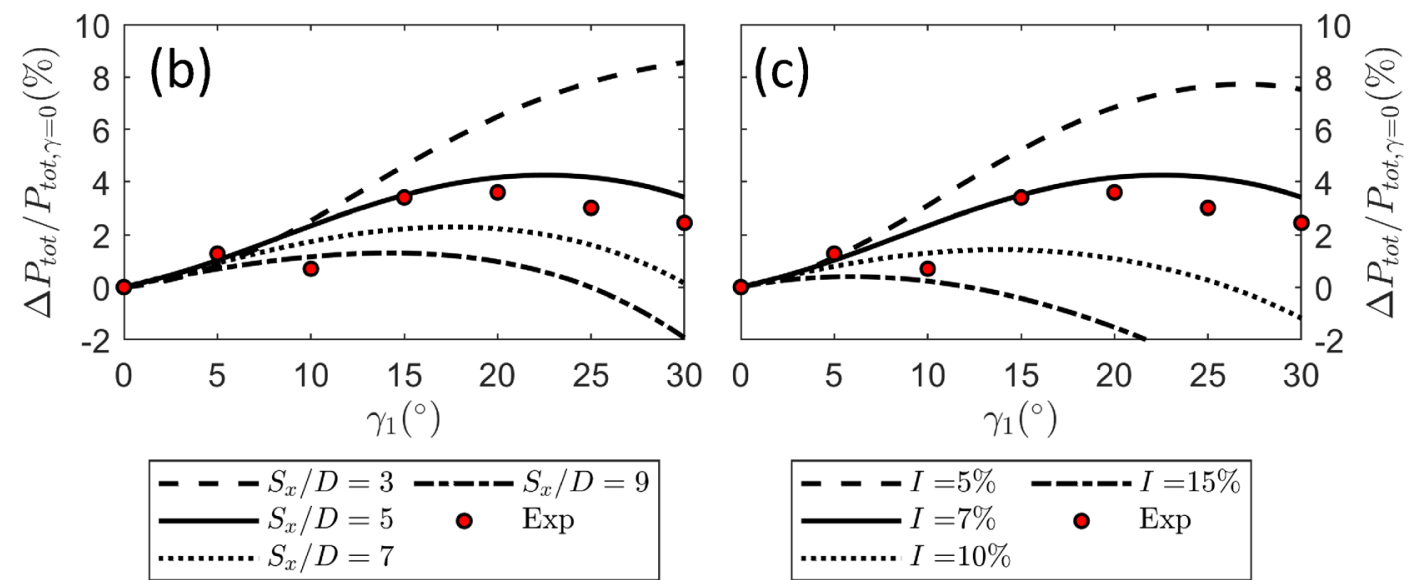

FIG. 11. (a) Measured power distribution of two turbines for different values of $\gamma_{1}$. (b) and (c) Variation of normalized total power increase (\%) with $\gamma_{1}$. Experimental data $\left(S_{x}=5 D\right.$ and $\left.I=7 \%\right)$ are shown with red circles, and the lines indicate analytical model predictions for different values of $S_{x}$ and $I$.

regardless of $n$. We therefore infer that there is a limit for the gain one could expect from yaw misalignment, and the linear relationship observed in Fig. 12(a) cannot be maintained for larger wind farms.

The wind farm studied experimentally in this paper consists of a single column of five wind turbines. In large wind farms with several columns of wind turbines, laterally deflected wakes might interact with adjacent columns, which can limit the gains obtained from yaw angle control. To shed light on the inter-
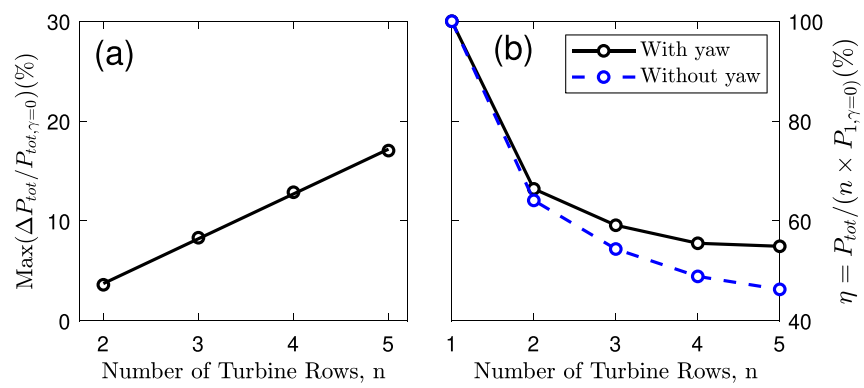

FIG. 12. (a) Normalized maximum wind farm power increase achieved by yaw angle control for different numbers of turbine rows. (b) Variation of wind farm efficiency $\eta$ with the number of turbine rows for wind farms with and without yaw angle control. column interaction in wind farms, the analytical wake model ${ }^{2}$ is used to visualize the border of the deflected wake for a yawed turbine. Figure 13 shows the wake flow distribution in a horizontal plane at hub height downwind of three side-by-side wind turbines, with a lateral spacing $S_{y}$ of $4 \mathrm{D}$. The turbine placed at $y=+4 \mathrm{D}$ has a yaw angle of $-30^{\circ}$, while the yaw angle for the other two is $+30^{\circ}$. The incoming turbulence intensity at hub height I is set to $7 \%$, which is the same as the one for the current experimental setup. In Fig. 13, note that only the values of normalized velocity deficit $\Delta \bar{u} / \bar{u}_{h}$ higher than 0.01 are shown in blue. As seen in the figure, the wakes of adjacent columns start interacting with each other roughly at $15 \mathrm{D}$ for those with the

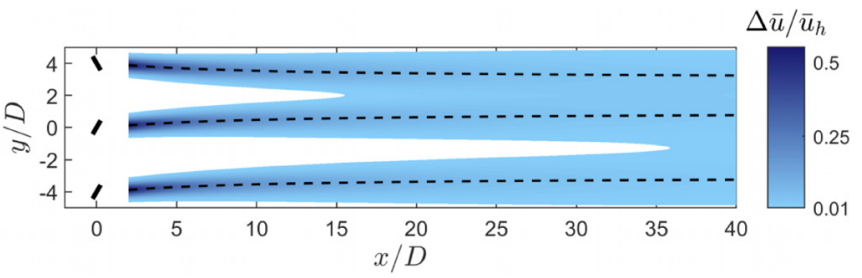

FIG. 13. Contours of the normalized streamwise velocity deficit, higher than 0.01 , in a horizontal plane at hub height downwind of three side-by-side yawed turbines. The turbine at $y=4 D$ has a yaw angle of $-30^{\circ}$, while the yaw angle for the other two is $+30^{\circ}$. 
opposite direction of the yaw angle and at 35D for those with the same direction of the yaw angle. These streamwise distances are equivalent to the fourth and eighth rows of a wind farm with $\mathrm{S}_{x}$ equal to $5 \mathrm{D}$, respectively. Based on this analytical analysis, one would therefore expect that inter-column interaction is insignificant for a wind farm consisting of five turbine rows, especially if wind turbines have the same direction of the yaw angle. However, this interaction should be taken into account for wind farms with a larger size and for those consisting of turbines with different directions of the yaw angle.

\section{SUMMARY AND FUTURE RESEARCH}

Wind tunnel experiments were performed to study the effect of yaw angle control on the overall efficiency of wind farms. To achieve this goal, the performance of a wind farm consisting of a column of five model wind turbines with a rotor diameter of $15 \mathrm{~cm}$ and a streamwise spacing of five rotor diameters was studied for a wide range of yaw angle distributions. The yaw angle and the rotational velocity of each turbine were monitored and controlled using electrical servo controllers. Special effort was made to ensure that all the turbines always operate at an optimal rotational velocity for any yaw angle and incoming flow condition. Power measurements were performed for 210 scenarios with different yaw angle distributions. All the yaw angle distributions tested in this study result in an overall wind farm power increase ranging from $0.5 \%$ to $17 \%$, with respect to the fully non-yawed conditions. Among the different yaw angle distributions, we were particularly interested in three strategies with different levels of simplicity and power improvement. In the first one, only the most upwind turbine operates in yaw. In the second strategy, all the turbines except for the last one have the same yaw angle. Finally, the third one concerns scenarios that maximize the wind farm efficiency with essentially any arbitrary yaw angle distribution. The results show that the increase in the overall wind farm efficiency by the simplest strategy (i.e., the first one) is relatively small (less than $4 \%$ for this experimental setup), but indeed not negligible. The second strategy can increase wind farm power more effectively (up to $12 \%$ for this setup) mainly by increasing the power generated by the last turbine. Finally, for the most successful scenarios in terms of overall wind farm efficiency (third strategy), one can observe that the first turbine has systematically a relatively large yaw angle, and the yaw angle value gradually decreases for subsequent rows until it eventually becomes zero for the last turbine. In general, the most optimal yaw angle distributions tend to homogenize the power distribution within the wind farm by decreasing the first turbine power and increasing the power generated by downwind turbines. We also investigated the suitability of yaw angle control for wind farms with different numbers of rows. The results show that, for the tested wind farms, the maximum achievable power enhancement via yaw angle control increases linearly with the number of turbine rows even though this increase is expected to reach an asymptotic value for large wind farms.

The results presented in this study point out that there is untapped potential to implement yaw angle control in utility- scale wind farms. There are, however, still some very important unexplored topics that have to be addressed in future studies:

- As seen earlier, large power degradation due to turbine wakes makes this wind farm a suitable candidate for implementing yaw angle control as a wake mitigation technique. It is therefore of interest to study the suitability of this technique for wind farms with other inflow conditions and layout configurations.

- In this work, the turbines are installed in a line parallel to the incoming flow. However, due to a continuous change of the wind direction in the field, it is quite common that the line on which wind turbines are installed is not parallel to the incoming flow direction (i.e., partial wake conditions). Therefore, yaw angle control of large wind farms under partial-wake conditions deserves more attention in future research.

- The effect of yaw misalignment on turbine loads is out of the scope of this study. However, this issue definitely merits further research in order to fully assess yaw angle control profitability given other technical aspects such as the turbine lifetime and maintenance costs.

- In this study, the turbines are only yawed in the same direction. However, as shown in previous research, ${ }^{2}$ both the magnitude and the direction of horizontal and vertical wake displacements under yawed conditions depend on the yawangle direction. Therefore, the effect of different yaw angle directions on the overall wind farm efficiency should be investigated in future studies.

- The detailed experimental dataset collected under fully controlled laboratory conditions in this study can provide useful qualitative insights into the suitability of yaw angle control for utility-scale wind farms. It is however important to note that the presented results cannot be generalized to the full scale in a quantitative manner due to a large difference in the Reynolds number that exists between the two cases. This therefore calls for future field measurements to quantitatively examine the effectiveness of yaw angle control in utility-scale wind farms.

\section{ACKNOWLEDGMENTS}

This research was supported by the Swiss National Science Foundation (Grant Nos. 200021-172538 and 206021144976), the Swiss Federal Office of Energy (Grant No. SI/ 501337-01), and the Swiss Innovation and Technology Committee (CTI) within the context of the Swiss Competence Center for Energy Research "FURIES: Future Swiss Electrical Infrastructure".

\section{APPENDIX: ADJUSTMENT OF TURBINE OPERATING CONDITIONS}

Figure 14 presents the algorithm for the adjustment and measurement of the turbines' performance from the beginning till the end of wind farm experiments. The matrix $\Gamma$ in the diagram contains all the information about yaw angle 


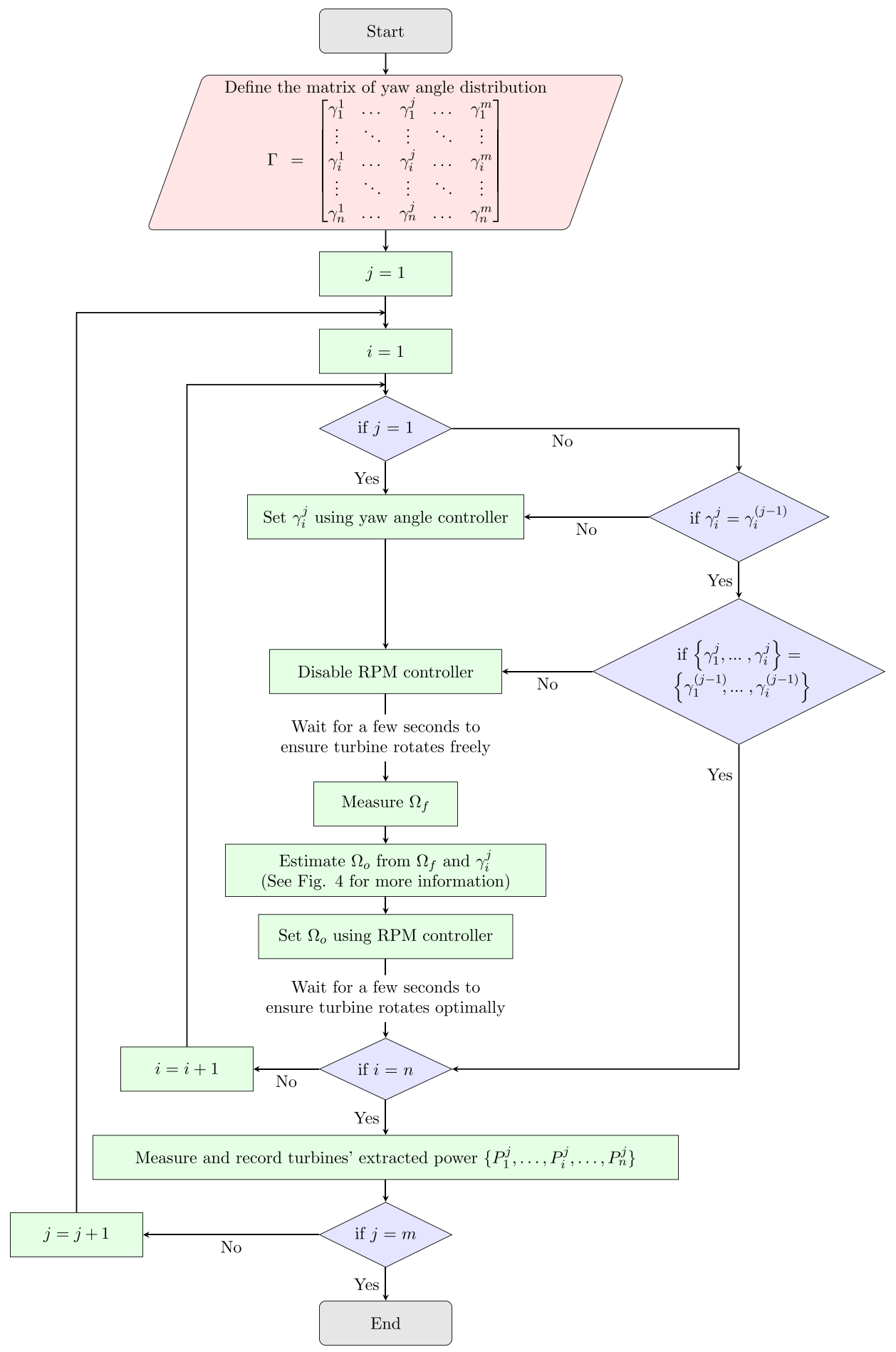

FIG. 14. Flowchart for the adjustment and measurement of the turbines' operating conditions for different yaw angle $\gamma$ distributions.

distributions tested in these experiments. Each row of the matrix corresponds to a scenario with a specific yaw angle distribution, while each column shows the yaw angle of a specific wind turbine for different scenarios. Therefore, the superscript $j$ denotes the scenario number ranging from 1 to $m$, where $m$ is the total number of tested scenarios $(m=210$ in this study), and the subscript $i$ denotes the turbine number ranging from 1 to $n$ ( $n=5$ in this study). 


\section{REFERENCES}

'Á. Jiménez, A. Crespo, and E. Migoya, "Application of a LES technique to characterize the wake deflection of a wind turbine in yaw," Wind Energy 13, 559-572 (2010).

${ }^{2} \mathrm{M}$. Bastankhah and F. Porté-Agel, "Experimental and theoretical study of wind turbine wakes in yawed conditions," J. Fluid Mech. 806, 506-541 (2016).

${ }^{3}$ H. Glauert, A General Theory of the Autogyro (HM Stationery Office, 1926).

${ }^{4}$ R. P. Coleman, A. M. Feingold, and C. W. Stempin, "Evaluation of the induced-velocity field of an idealized helicoptor rotor," Technical Report No. L5E10, DTIC Document, 1945.

${ }^{5}$ I. Grant, P. Parkin, and X. Wang, "Optical vortex tracking studies of a horizontal axis wind turbine in yaw using laser-sheet, flow visualisation," Exp. Fluids 23, 513-519 (1997).

${ }^{6}$ I. Grant and P. Parkin, "A DPIV study of the trailing vortex elements from the blades of a horizontal axis wind turbine in yaw," Exp. Fluids 28, 368-376 (2000).

${ }^{7} \mathrm{D}$. Medici and P. Alfredsson, "Measurement on a wind turbine wake: 3D effects and bluff body vortex shedding," Wind Energy 9, 219-236 (2006).

${ }^{8}$ P. A. Fleming, P. M. O. Gebraad, S. Lee, J. van Wingerden, K. Johnson, M. Churchfield, J. Michalakes, P. Spalart, and P. Moriarty, "Evaluating techniques for redirecting turbine wakes using SOWFA," Renewable Energy 70, 211-218 (2014).

${ }^{9}$ M. F. Howland, J. Bossuyt, M.-T. L. A. J. Meyers, and C. Meneveau, "Wake structure in actuator disk models of wind turbines in yaw under uniform inflow conditions," J. Renewable Sustainable Energy 8, 043301 (2016).

${ }^{10} \mathrm{M}$. Churchfield, Q. Wang, A. Scholbrock, T. Herges, T. Mikkelsen, and M. Sjöholm, "Using high-fidelity computational fluid dynamics to help design a wind turbine wake measurement experiment," J. Phys.: Conf. Ser. 753, 032009 (2016).

"J. Wang, S. Foley, E. Nanos, T. Yu, F. Campagnolo, C. Bottasso, A. Zanotti, and A. Croce, "Numerical and experimental study for wake redirection techniques in a boundary layer wind tunnel," J. Phys.: Conf. Ser. 854, 012048 (2017).

${ }^{12}$ C. R. Shapiro, D. F. Gayme, and C. Meneveau, "Modelling yawed wind turbine wakes: A lifting line approach," J. Fluid Mech. 841, R1 (2018).

${ }^{13}$ J. Schottler, J. Bartl, F. Mühle, L. Sætran, J. Peinke, and M. Hölling, "Wind tunnel experiments on wind turbine wakes in yaw: Redefining the wake width," Wind Energy Sci. 3, 257-273 (2018).

${ }^{14}$ D. Medici, S. Ivanell, J.-A. Dahlberg, and P. H. Alfredsson, "The upstream flow of a wind turbine: Blockage effect," Wind Energy 14, 691-697 (2011).

${ }^{15} \mathrm{M}$. Bastankhah and F. Porté-Agel, "Wind tunnel study of the wind turbine interaction with a boundary-layer flow: Upwind region, turbine performance, and wake region," Phys. Fluids 29, 065105 (2017).

${ }^{16}$ A. Ozbay, W. Tian, Z. Yang, and H. Hu, "Interference of wind turbines with different yaw angles of the upstream wind turbine," in 42nd AIAA Fluid Dynamics Conference and Exhibit (2012), p. 2719.
${ }^{17} \mathrm{P}$. Krogstad and M. S. Adaramola, "Performance and near wake measurements of a model horizontal axis wind turbine,"Wind Energy 15, 743-756 (2012).

${ }^{18} \mathrm{M}$. Bastankhah and F. Porté-Agel, "A wind-tunnel investigation of windturbine wakes in yawed conditions," J. Phys.: Conf. Ser. 625, 012014 (2015).

${ }^{19}$ J. Bartl, F. Mühle, J. Schottler, L. Sætran, J. Peinke, M. Adaramola, and M. Hölling, "Wind tunnel experiments on wind turbine wakes in yaw: Effects of inflow turbulence and shear," Wind Energy Sci. 3, 329-343. (2018).

${ }^{20} \mathrm{M}$. Adaramola and P.- $\AA$. Krogstad, "Experimental investigation of wake effects on wind turbine performance," Renewable Energy 36, 2078-2086 (2011).

${ }^{21}$ P. M. O. Gebraad, F. W. Teeuwisse, J. W. Wingerden, P. A. Fleming, S. D. Ruben, J. R. Marden, and L. Y. Pao, "Wind plant power optimization through yaw control using a parametric model for wake effects-a CFD simulation study," Wind Energy 19, 95-114 (2016).

${ }^{22}$ J. Park and K. H. Law, "A data-driven, cooperative wind farm control to maximize the total power production," Appl. Energy 165, 151-165 (2016).

${ }^{23}$ J. Park and K. H. Law, "Bayesian ascent: A data-driven optimization scheme for real-time control with application to wind farm power maximization," IEEE Trans. Control Syst. Technol. 24, 1655-1668 (2016).

${ }^{24}$ F. Campagnolo, V. Petrović, J. Schreiber, E. M. Nanos, A. Croce, and C. L. Bottasso, "Wind tunnel testing of a closed-loop wake deflection controller for wind farm power maximization," J. Phys.: Conf. Ser. 753, 032006 (2016).

${ }^{25}$ P. A. Fleming, J. Annoni, M. J. Churchfield, L. Martinez, K. M. Gruchalla, M. J. Lawson, and P. J. Moriarty, "A simulation study demonstrating the importance of large-scale trailing vortices in wake steering," Wind Energy Sci. 3, 243-255 (2018).

${ }^{26}$ J. M. S. Bartl, F. V. Mühle, and L. R. Sætran, "Wind tunnel study on power output and yaw moments for two yaw-controlled model wind turbines," Wind Energy Sci. 3, 489 (2018).

27J. R. Garratt, "Review: The atmospheric boundary layer," Earth-Sci. Rev. 37, 89-134 (1994).

${ }^{28}$ R. B. Stull, An Introduction to Boundary Layer Meteorology (Springer Science, 2009), Vol. 13.

${ }^{29} \mathrm{M}$. Bastankhah and F. Porté-Agel, "A new miniature wind turbine for wind tunnel experiments. Part i: Design and performance," Energies 10, 908 (2017).

${ }^{30} \mathrm{M}$. Bastankhah and F. Porté-Agel, "A new miniature wind turbine for wind tunnel experiments. Part ii: Wake structure and flow dynamics," Energies 10, 923 (2017).

${ }^{31}$ R. Barthelmie, G. C. Larsen, S. T. Frandsen, L. Folkerts, K. Rados, S. Pryor, B. Lange, and G. Schepers, "Comparison of wake model simulations with offshore wind turbine wake profiles measured by sodar," J. Atmos. Oceanic Technol. 23, 888-901 (2006).

${ }^{32}$ F. Carbajo Fuertes, C. D. Markfort, and F. Porté-Agel, "Wind turbine wake characterization with nacelle-mounted wind lidars for analytical wake model validation," Remote Sens. 10, 668 (2018).

${ }^{33}$ D. Dilip and F. Porté-Agel, "Wind turbine wake mitigation through blade pitch offset," Energies 10, 757 (2017). 